\title{
CONTROL STRATEGIES FOR A MULTIPLE DEGREE OF FREEDOM PROSTHETIC HAND
}

\author{
D.P.J. Cotton, A. Cranny, P.H. Chappell, N.M. White, S.P. Beeby \\ Electronic Systems Design Group, School of Electronics and Computer Science, \\ University of Southampton, Southampton, S017 1BJ, UK
}

\begin{abstract}
Some of the traditional methods used to control a conventional prosthetic device are described alongside the current state of new control techniques and how they may progress. The review includes implantable myoelectric sensors and describes the potential of connecting directly to the peripheral nervous system. Control methods are then deduced for each technique, where the application is a six degrees of freedom hand having integral slip, force and temperature sensors.
\end{abstract}

Keywords: Control strategy, prosthetic hand, implantable sensors.

\section{INTRODUCTION}

The human hand is complex. It has 27 bones and a multitude of muscles and tendons to provide a large number of degrees of freedom in movement. In addition, each hand has an array of over 17000 tactile sensors. With existing technology it is near impossible to replicate anything mechanically similar. However, advances in technology have enabled some considerable improvements in the functionality of a prosthetic hand with an increase in the number of degrees of freedom available through the use of smaller and lighter motors. There are some compromises made though, with the maximum grip force being reduced. However, with a more adaptive grasp less force is required to hold an object, allowing the use of less powerful motors. With this increase in available mechanical functionality come new challenges of control.

\subsection{Conventional Prosthetic hands}

At present, commercially available prosthetic hands are limited to a single degree of freedom (DOF) in their movement. Traditionally a single motor drives the first and second fingers of the hand in unison with the thumb to produce a tri-digital grip. The remaining two fingers on the hand are for aesthetic purposes and move passively with the first two fingers. Two good examples are the Otto Bock SensorHand ${ }^{\mathrm{TM}}$ Speed (Otto Bock, 2006) and the Motion Control Hand (2006).

The control inputs of a prosthetic hand can be limited to a single myoelectric input from a muscle pair within the lower arm. For patients with higher amputations or who wish to have more flexibility, such as a wrist rotator, extra control inputs can be provided such as proportional control switches or pressure switches activated by muscle bulge.
However control of the prosthesis using multiple inputs is not intuitive and could require long periods of training to obtain satisfactory use. A good overview of the commercially available inputs for prosthetics can be found in Otto Bock (2005).

\subsection{The Southampton REMEDI Hand}

The Southampton REMEDI hand (Light 2000; Light and Chappell 2000) has six degrees of freedom and is shown in Figure 1. The hand consists of six small electrical motors, two of which are used to actuate the extension-flexion, and rotation movements of the thumb with each of the remaining four motors being assigned to individual fingers. Each finger is made from six bar linkages, which when extended or flexed curl in a fixed anthropomorphic trajectory (Guo and Gruver 1993). To reduce the power required to hold an object, the fingers are driven via a worm wheel gear configuration. This also has the additional advantage that it prevents the fingers being back driven after power is removed from the motor. The drive from the worm wheel configuration also increases the torque produced from the small motors to provide a $9 \mathrm{~N}$ grip force at the end of each finger. Located on each fingertip is an array of thick-film sensors which can be used to monitor the force exerted by the fingertip as well as detect the onset of object slip and measure temperature (Cranny et al 2005 a,b).

A similar multiple DOF hand called the iLimb Hand $^{\mathrm{TM}}$ developed by Touch Bionics (2006) is currently under development and should be commercially available in the near future. 


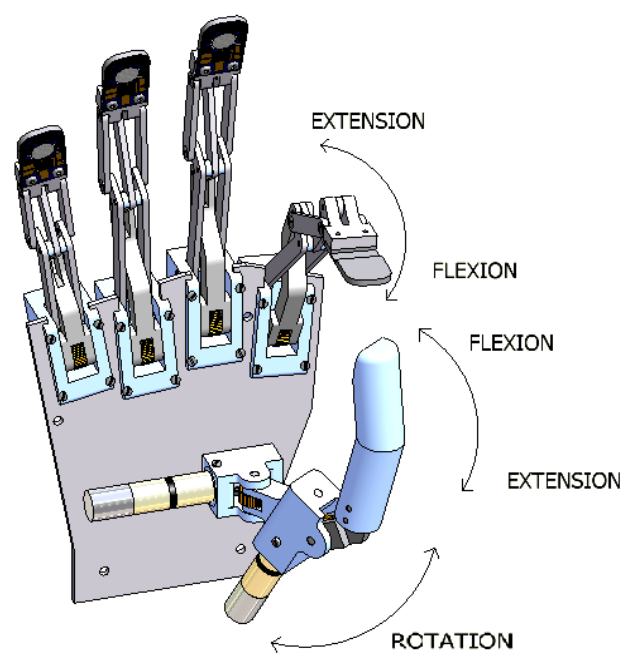

Fig. 1: Computer generated model of the Southampton REMEDI hand.

\subsection{Control of the Southampton REMEDI hand}

In order to utilise the increased functionality of the Southampton REMEDI hand a hybrid controller using the Southampton adaptive manipulation scheme, SAMS (Nightingale 1985), in conjunction with the UNB MyoController (Hudgins 1993) has been proposed (Light et al 2002).

Myoelectric signals are produced for different attempted movements of the arm, for example extending the elbow or flexing the elbow, exhibit different characteristics from the muscles. The UNB MyoController uses these signals from a muscle pair and classifies them. Each separate classified signal can then be used to control a different function within the prosthesis such as a wrist rotation or the opening and closing of the hand. Using this technique up to four movement types can be realised from an extensor/ tensor muscle pair.

The SAMS controller illustrated in Figure 2 is constructed of a number of states which are used during object manipulation. The POSITION state allows the user to adopt the required prehensile pattern. The prosthesis is then set in a voluntary open, involuntary close mode. Therefore in order to hold the hand open extensor muscle activity must be detected. The hand opens proportionally to the amplitude of the signal detected. If no signal is detected then the hand will close until the force sensors detect contact with an object. At this point the hand will stop and adopt the TOUCH state with a minimum grip force applied to the object. A further flexion EMG signal will then move the controller on to the HOLD mode where the slip detectors are activated and automatically control the grip force to prevent the object from slipping out of the prosthesis. The user may then override the controller with a flex of the muscle to increase grip force in the SQUEEZE mode or just simply release the object with an extend EMG signal.

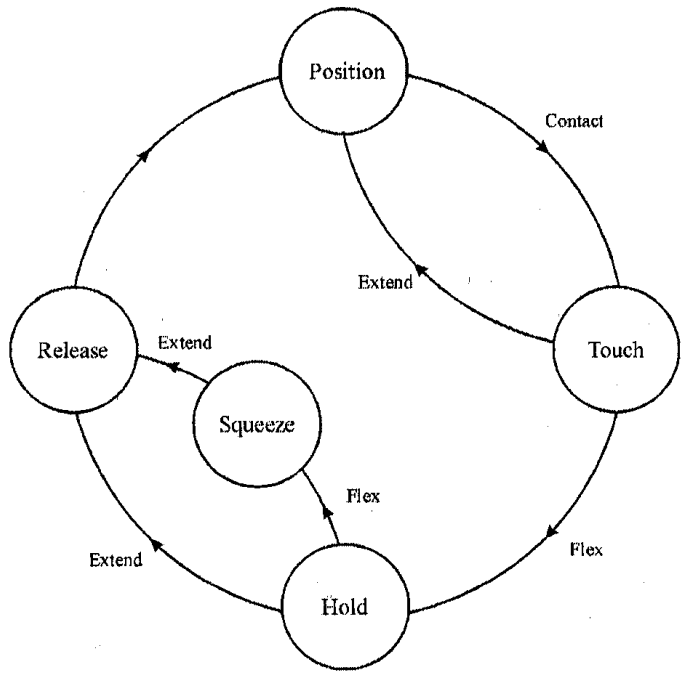

Fig. 2: Southampton adaptive manipulation scheme (SAMS) state diagram.

Combining the UNB MyoController and the SAMS controller allows the user to initially select one of four pre-programmed prehensile grip patterns as determined by the UNB MyoController from the user's signal. From this stage onwards the SAMS hierarchical control system is activated and any change of grip pattern from the controller is ignored. At any stage during the grasping process a maintained period of extensor activity will reset the controller to the initial grip pattern select mode.

\section{DIRECT CONNECTION TO THE PERIPHERAL NERVOUS SYSTEM}

To connect a prosthetic hand to the peripheral nervous system is considered extremely advantageous by many researchers in the field of prosthetics. Essentially, the original control inputs from the amputated hand are being used to control a device for the same purposes. This would provide a natural feeling control to take place with minimum cognition experienced by the user and potentially very little training would be required to use the device.

Recent work in the UK has investigated implanting a $4 \mathrm{~mm} \times 4 \mathrm{~mm}, 100$ element electrode array into the median nerve of an able bodied male (Gasson et al 2005). A threshold was applied to the output signals obtained from the electrode array to control the opening and closing of a multiple degree of freedom hand operating under the SAMS control system. The hand (Kyberd et al 1998) also incorporated force sensors on the fingertips, which were used to provide feedback directly to the peripheral nerves as a current in the range 80-100 $\mu \mathrm{A}$. Experiments were undertaken using visual feedback, force feedback and a combination of both to grip an object as lightly as possible. The results showed that using a combination of both visual and force feedback allowed the user to provide the lightest grip whereas using visual feedback or force feedback on their own showed that considerably higher grip forces where applied. The implant was successfully removed after 
96 days showing no signs of infection to the subject. Post analysis of the implant indicated little evidence of rejection by the body. This suggests that this type of implant could be available for long term use in the future.

Recent research in the USA by Dhillon et al (2005) has involved implanting electrodes directly into the fascicles of severed nerves in six upper limb amputees. Trials were then carried out over a period of six days using feedback to the nerves to control the grip force of a prosthetic hand. A similar test using feedback to the nerves to indicate the elbow joint position of a prosthetic arm was also carried out. Both tests used only a single input and output and no visual feedback from the user was allowed. The results are promising and demonstrate that good motor control of the prosthesis with direct feedback to the nerves can be obtained.

More research is therefore required to target specific motor functions and this technique is not likely to be available for general use in the near future. However, these initial results show that direct control inputs can be harnessed from the peripheral nerves and tactile sensor information successfully returned to the user, which looks promising for the use of returning natural hand control to a patient.

The Defense Advanced Research Projects Agency (DARPA) in the USA has recently received government funding to investigate creating a new multiple DOF hand and the potentials of different control methods, specifically concentrating on neural control from the peripheral nervous system (DAPRA 2006). This is in direct response to over 200 new amputees as a result of the recent conflicts in Iraq and Afghanistan. The 48 month project is well funded and aims to drive forward all aspects of upper limb prosthetics design and control with the ultimate goal to produce an easily controlled multiple DOF hand.

\subsection{Proposed control system}

It is proposed that each motor of the Southampton REMEDI hand will be controlled independently so that the user will easily be able to define their own prehensile grip patterns. The signals provided from the nerves will be proportional to the speed at which the fingers move until TOUCH is detected, at which point the signal will become proportional to the applied grip force. Once the force has remained approximately constant for a period of time the HOLD function will be activated to prevent object slip. This will replicate the automatic control of the grip force when slip has occurred in the human hand and help to reduce the cognition on the user through visual monitoring of the object. The force, slip and motor encoders will also be used to send tactile feedback to the user.

\section{IMPLANTABLE MYOELECTRIC SENSORS (IMES)}

Surface electromyography (EMG) sensors are currently the sensor of choice in controlling upper limb prosthetics. They pick up the EMG signals from a number of muscles and combine them together depending on where they are placed. Recently Weir et al (2003) have reported on using implantable myoelectric sensors to target specific muscles in the arm which are used to control arm function. There are a range of muscles contained in the human arm and by targeting muscles used for specific operations in the lost limb, a more natural feel to hand control will be achieved. The number of sites available for use to control the prosthesis will ultimately depend upon the level of amputation for each individual patient. An initial concept test has shown that six EMG signals can be successfully obtained from the arm. When fully developed each wireless IMES sensor will be injected into a target muscle and the signals sent to an external controller via a transcutaneous magnetic link. The sensors will be powered using an external magnetic field. Currently work is still required to reduce the size of the external reader coil to fit within a prosthesis socket. The power consumption of the reader must also be addressed to allow a suitably sized battery to be fitted within the prosthesis.

A similar project involving injecting $\mathrm{BION}^{\mathrm{TM}}$ electrical stimulators into a muscle to stimulate specific targeted muscles in stroke patients has been used to provide better upper limb control (Notley 2005). The IMES sensors will use the same packaging and implant method as used in this technique except instead of stimulating the muscles they will be used to detect the signals from them.

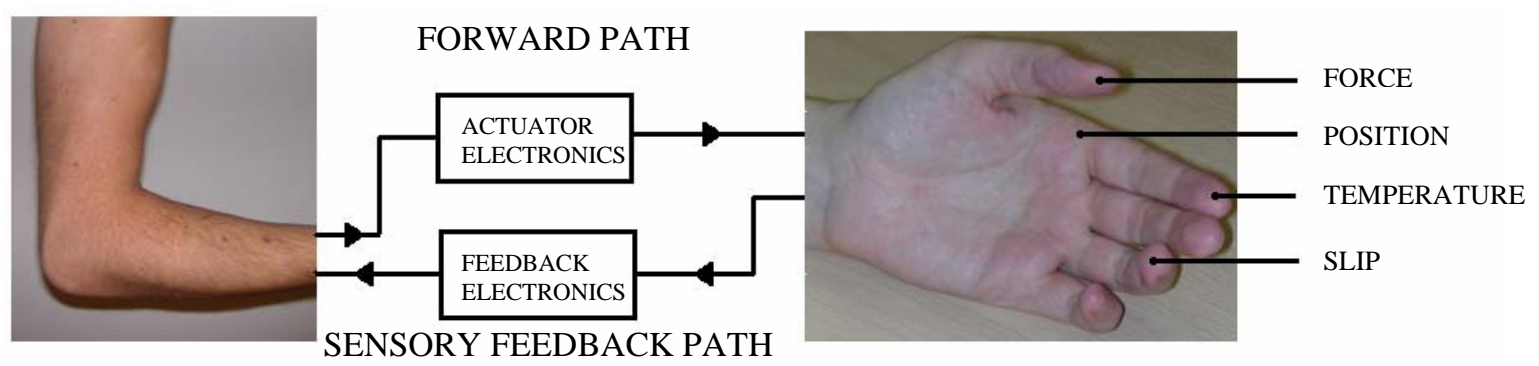

Fig. 3: Control system using direct inputs from the peripheral nervous system. 


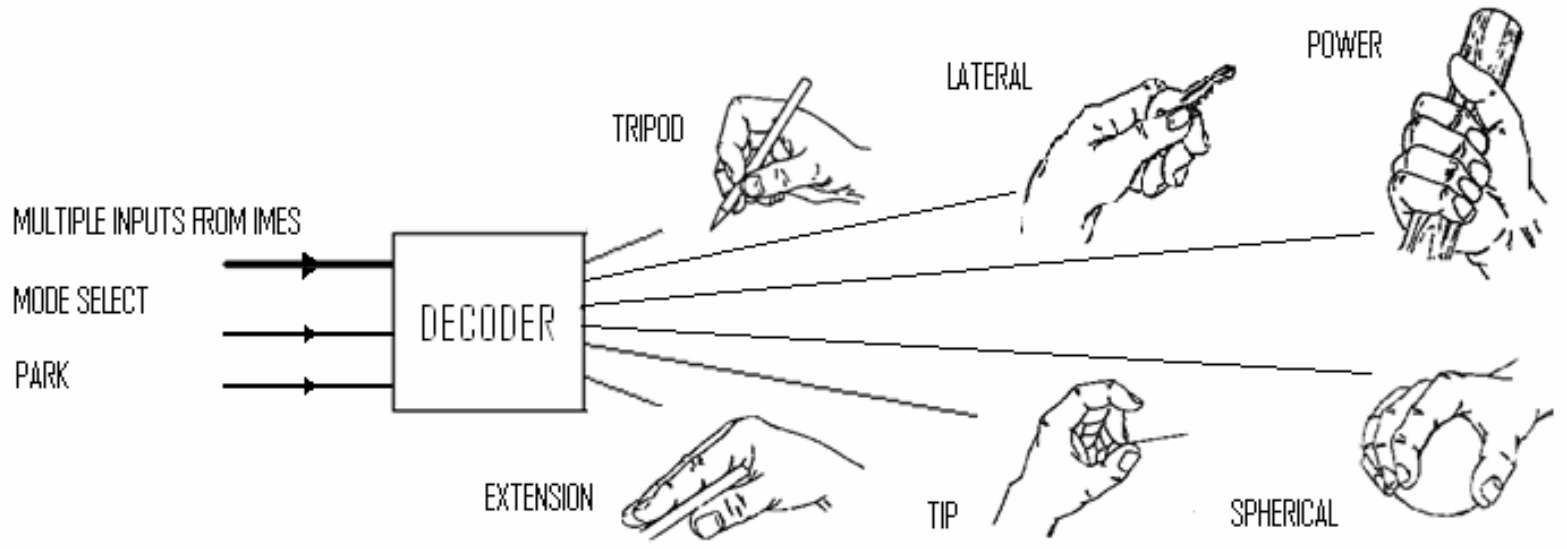

Fig. 4: Potential control system using IMES inputs with six useful prehensile grip patterns.

It is predicted that this technology will enable the user to control 8 DOF with more than one degree of freedom being controlled simultaneously. However it is not clear how easy this control would be when producing some types of grip. With this in mind the following control system is proposed for a possible 8 DOF input.

Six of the 8 inputs would be used to select the prehensile grip pattern or an independent finger/ thumb movement. When a pattern has been selected the same input signal will be utilised to proportionally control the grip force applied to the object. Once the user stops producing this signal the hand enters a HOLD state as defined in the SAMS controller. The user then has three options: they can override the HOLD state to apply a SQUEEZE to the object using the same input; reset the hand to return to a fully open rest position (using the PARK input) or reset the hand using one of the five other prehensile patterns using a single EMG burst. This would then set up the hand into another prehensile pattern ready for its next task.

A single input (MODE SELECT) would be used to disengage the SAMS controller and allow individual fingers or the thumb to be moved independently, or more prehensile patterns to be produced depending upon the users preferences.

\subsection{Typical grip postures.}

There are a number of different grip postures which we all use in our activities of daily living (ADL). These postures are naturally adopted before the task begins allowing the most efficient manner of undertaking a task to be achieved. For example the lateral grip is often used for unlocking a door with a key. However you would probably not use this type of grip when lifting a heavy object.

The six prehensile grip postures illustrated in Figure 4 would allow an amputee the freedom to undertake the majority of ADL in a much more natural way than is currently allowed by a single degree of freedom tripod grip.

\section{ALTERNATIVE UPPER LIMB CONTROL INPUTS}

\subsection{Insole foot controller}

Carrozza et al (2005) have reported the development of a controller that uses selected foot movements to actuate a robotic hand. The prototype device is based around a standard shoe insole which has four purpose built pressure switches attached to it. The switches are located in four different areas, under the big toe, the heel and two at the sides of the foot. The battery and electronics are also contained within the insole under the arch of the foot. To transmit the signals to the hand Bluetooth wireless technology is used. There is also an on/off switch incorporated into the insole in the form of an accelerometer, so by tapping the shoe on the floor the pressure switches can be activated or deactivated. This is essential to remove control of the hand during other tasks such as walking. To test the ease of use of the foot controller 10 able bodied subjects were fitted with the device and a 9 DOF hand (Carrozza et al 2003) attached to the end of the subjects hand. They were then trained to undertake some simple tasks using the ACHILLE foot controller and using EMG signals as a comparison to control the arm. The results showed that the foot controller was much easier to learn how to use than the EMG controller.

\subsection{Targeted muscle hyper-reinnervation}

Targeted muscle hyper-reinnervation is a technique developed by Kuiken et al (1995) investigated in rats. It involves sewing large nerves containing many motor neurons onto a small muscle area to hyperreinnervate the muscle. This then allows the muscle to be controlled using signals from the transferred nerve.

In an upper limb amputee the brachial plexus nerves, which control the muscles in the arm, become severed. There are four nerves which make up the brachial plexus nerves. These are the ulna, radial, median and musculocutaneous, and each controls different muscle groups within the arm. The number of nerve 
reinnervations available depends on the level of amputation in the patients arm. For a wrist amputation the median and ulna nerve may be used to create two new EMG sites as they both control some of the muscles in the hand (Moore and Dalley 2006). However to use the musculocutaneous and ulna nerves would mean loosing function from other muscles unless the patient had suffered a higher amputation. The advantage of using these new EMG sites over other sites is that the patient feels as though they are actually using their hand rather than activating a muscle which has not previously been associated with a hand or arm movement.

An operation on a bilateral shoulder disarticulation amputee (Kuiken et al 2004) has proved muscle hyper-reinnervation to be successful in humans. The patient had all four brachial plexus nerves reinnervated onto a segmented pectoralis major muscle and the pectoralis minor. After surgery the patient was instructed to simulate using their arm and hand muscles everyday to stimulate the nerves and help reinnervate the muscles. It took approximately 3 months before the first signs of muscle twitching occurred and approximately 5 months before 3 out of 4 reinnervated muscles were active. Some of the sensors in the chest were also reinnervated and the patient reported feeling parts of his amputated hand and arm when certain parts of his chest were touched. The three new EMG sites were subsequently used to control a prosthetic arm.

\section{CONCLUSIONS}

Future developments in harnessing natural control inputs from a subject who has suffered the loss of a hand are an essential factor in enabling the full potential of a multifunctional prosthetic hand to be realised. Developments in harnessing information from the peripheral nervous system and particularly IMES both show the potential in the future to provide these extra natural control inputs. However, even with these extra control inputs there is still a need for some automation within the actuation of the prosthesis as found in the human hand. Examples of this include an automatic increase in grip force when slip is detected or the rejection of an object when a high temperature which may damage the prosthesis is detected.

Any new control algorithm for the control of a prosthetic hand using new input technology will have to be adaptive for different patients to take into account the level of amputation as well as personal preferences of control. Nevertheless, some of the original SAMS control states may still prove to be extremely useful in reducing the cognitive strain on the user.

\section{REFERENCES}

Cranny, A Cotton, DPJ Chappell, PH Beeby,SP and White, NM (2005) Thick-film force, slip and temperature sensors for a prosthetic hand, Measurement Science and Technology 16, 111.

Cranny, A Cotton, DPJ Chappell, PH Beeby, SP White, NM Thick-film force and slip sensors for a prosthetic hand, Sensors and Actuators A (Physical) 123-124, 162-171.

Carrozza, MC Dario, P Vecchi, F Roccella, S Zecca, M Sebastiani, F (2003) The Cyberhand: on the design of a cybernetic prosthetic hand intended to be interfaced to the peripheral nervous system Proceedings.2003 IEEE/RSJ International Conference on Intelligent Robots and Systems (IROS 2003), 2642-2647.

Carrozza, MC Persichetti, A Laschi, C Vecchi, F Vacalebri, P Tamburrelli, V Lazzarini, R and Dario, P (2005) A novel wearable foot interface for controlling robotic hands, 2005 IEEE/RSJ International Conference on Intelligent Robots and Systems (IROS 2005), 2010 - 2015.

DARPA http://www.darpa.mil/dso/solicitations/ prosthesisPIP.htm [accessed 3/4/06]

Dhillon, GS and Horch, KW (2005) Direct neural sensory feedback and control of a prosthetic arm, IEEE Transactions on Neural Systems and Rehabilitation Engineering 13, 468-472.

Gasson, M Hutt, I Kyberd, P and Warwick, K (2005) Invasive neural prosthesis for a neural signal detection International Journal of Adaptive Control and Signal Processing 19, 365-375.

Hudgins, B Parker, P and Scott, RN (1993) A new strategy for multifunctional myoelectric control. IEEE Transactions on Biomedical Engineering 40, 82-84.

Kuiken, TA Childress, DS and Rymer, WZ (1995)The hyper-reinnervation of rat skeletal muscle, Brain Surgery 676, 113-123.

Kuiken, TA Dumanian, G.A Lipschutz,R. D Miller, L.A Stubblefield, K.A (2004) The use of targeted muscle reinnervation for improved myoelectric prosthesis control in a bilateral shoulder disarticulation amputee, Prosthetics and Orthotics International 28(3), 245-253.

Kyberd, PJ, Evans, M and Winkel, S (1998) An intelligent anthropomorphic hand with automatic grasp, Robotica, 16, 531-536.

Light, CM (2000) An intelligent hand prosthesis and evaluation of pathological and prosthetic hand function. PhD Thesis, University of Southampton.

Light, CM and Chappell, PH (2000) Development of a light weight and adaptable multiple axis hand prosthesis, Medical Engineering and Physics, 22, 679-684.

Light, CM Chappell, PH Hudgins, B and Engelhart, K (2002) Intelligent myoelectric control of hand prosthesis, Journal of Medical Engineering and Technology, 26 (4), p139-146.

Nightingale, JM (1985) Microprocessor control of an artificial arm, Journal of Microcomputer Applications 8, 167-173. 
Notley SV, Turk R, Burridge JH, Cosendai G, and Ripley AM (2005) Sensors for Open Loop Control of Reaching and Grasping using the BION Microstimulators, 10th Annual Conference of the International Functional Electrical Stimulation Society (IFESS).

Moore, KL and Dalley, AF (2006) Clinically orientated anatomy fifth edition ISBN 0-78173639-0.

Motion Control http://www.utaharm.com/tds.htm [accessed 6/4/06].

Otto Bock (2005) MYOBOCK arm components catalogue 2005.

Otto Bock www.ottobock.co.uk [accessed 3/4/06]

Touch Bionics www.touchbionics.com [accessed 3/4/06].

Weir, RFff Troyk, PR DeMicele, G and Kuiken, T (2003) Implantable myoelectric sensors (IMES) for upper-extremity prosthesis control preliminary work, Proceedings of the $25^{\text {th }}$ International Conference of the IEEE EMBS Cancun, Mexico September 17-21, 1562-1564. 